\title{
A SIMPLE AND EFFECTIVE ELEMENT FOR ANALYSIS OF GENERAL SHELL STRUCTURES
}

\author{
KLAUS-JÜRGEN BATHE and LEE-WING Ho \\ Department of Mechanical Engineering. Massachusetts Institute of Technology. Cambridge, MA 02139, U.S.A.

\begin{abstract}
A simple flat three-node triangular shell element for linear and nonlinear analysis is presented. The element stiffness matrix with 6 degrees-of-freedom per node is obtained by superimposing its bending and membrane stiffness matrices. An updated Lagrangan formulation is used for large displacement analysis. The application of the element to the analysis of various linear and noninear problems is demonstrated.
\end{abstract}

\section{IMTronuction}

Two approaches have basically been employed in the recent efforts on the development of general shell analy. sis capabilities [1, 2]:

- High-order isoparametric elements based on degenerating fully three-dimensional stress conditions have been proposed.

- Low-order simple elements that are basically obtained by superimposing plate bending and membrane stiffnesses have been developed.

The higher-order isoparametric elements are very versatile (they can be employed as transition elements $[2,3]$ ) and are quite effective, but they can be costly in use. The element stiffness matrix is relatively large in size and a sufficiently high enough integration order must be used to avoid the introduction of spurious zero energy modes.

The premise of the simple low-order elements lies in that their related matrices can be formed inexpensively. Thus, even when a large number of elements are required to model a complex structure, the overall analysis effort may still be less than with the use of the higher-order isoparametric shell elements. Also, the direct use of stress resultants (moments, membrane forces) may not only decrease the cost of analysis, but also facilitates the interpretation of the computed results.

Various simple low-order elements have been proposed recently [4-8]. When evaluating these elements for practical analysis, we believe that the following three criteria should be considered:

(1) The element should yield accurate solutions when modeling any shell geometry and under all boundary and loading conditions. In particular, the element should exactly contain the required 6 zero rigid modes, so that reliable results can always be expected. The theory of the element formulation must be well-understood and should not contain any "numerical fudge factors".

(2) We should be able to use the element in the modeling of general shell structures with beam stiffeners, cut-outs, intersections, and so on.

(3) The element should be cost-effective in linear as well as in nonlinear, static and dynamic analysis. In nonlinear analysis, the element should be applicable to large displacement, large rotation, and materially nonlinear conditions.

Considering the above criteria we want to emphasize that the reliability aspect in (1) is the most important. Yet, a considerable number of elements that have been published do not satisfy this criterion. Such element developments represent interesting research, but should not be used in actual engineering analyses, because the generated analysis results cannot be interpreted with confidence.
The objective in this paper is to present a shell element that is simple and effective and that has been developed with due regard to the above requirements. The element is shown schematically in Fig. 1. We observe that the element is flat and has 3 nodes with 6 degrees of freedom per node. The total element stiffness matrix is formulated by superimposing-in the way some of the earliest shell elements were formulated [9]-a plane stress membrane stiffness $\mathbf{K}_{N,}$ a bending stiffness $\mathbf{K}_{B}$ and an in-plane rotational stiffness $\mathbf{K}_{\theta_{\text {i }}}$. In the next section we discuss the derivation of these stiffness matrices for linear analysis. The updated Lagrangian formulation used in large displacement analysis is then presented in Section 3 and finally in Section 4 we present the results obtained in various demonstrative sample analyses.

\section{FORMULATION OF ELBMANT TOR DFNTTESMAL DISPLACRMENT ANALYSIS}

Since the complete stiffness matrix of the element is obtained by the direct superposition of $\mathbf{K}_{M}, \mathbf{K}_{B}$ and $\mathbf{K}_{\theta_{z}}$ we can discuss the formulation of these matrices independently.

\subsection{Membrane stiffness matrix}

The element membrane stifiness $\mathbf{K}_{M}$ is simply the constant strain plane stress stiffness matrix of a 3 -node element.

\subsection{Bending stiffness matrix}

The bending stiffness matrix is formulated using the Mindlin theory of plates with shear deformations included. Using the variables defined in Fig. 2, the displacement components of a point with coordinates $x, y, z$ are in this theory $[8]$

$$
u=z \beta_{x}(x, y) ; v=-z \beta_{y}(x, y) ; \text { and } w=w(x, y)
$$

where $w$ is the transversal displacement, $\beta_{x}$ and $\beta_{y}$ are the rotations of the normal to the undeformed middle surface about the $y$ and $x$ axes, respectively.

The bending strains vary linearly through the thickness

$$
\epsilon_{b}=2 \kappa
$$

where $\boldsymbol{x}$ is the three component vector of curvatures

$$
\kappa=\left[\begin{array}{c}
\beta_{x, x} \\
-\beta_{y, y} \\
\beta_{x, y}-\beta_{y, x}
\end{array}\right]
$$



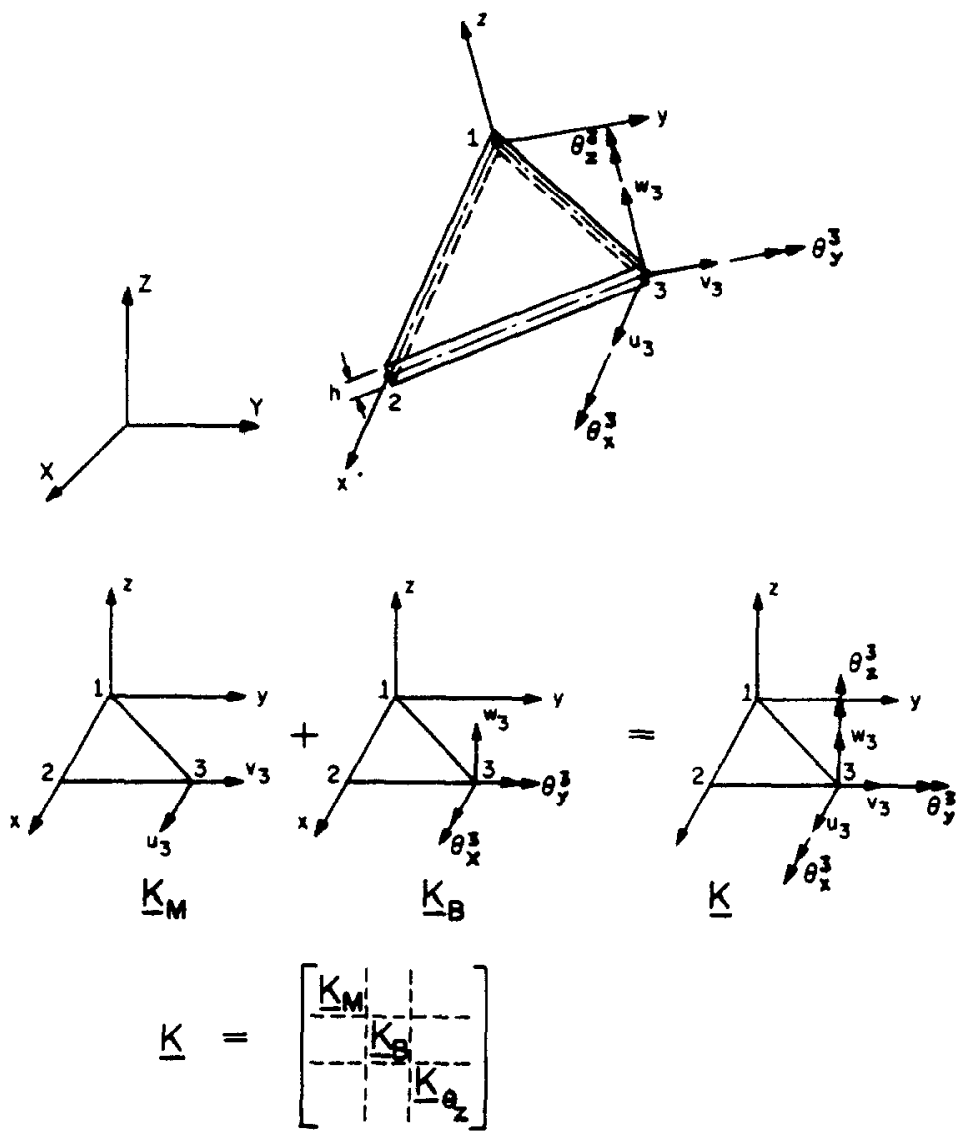

Fig. I. The sheil element.

and the transverse shear strains are constant through the thickness,

$$
\gamma=\left[\begin{array}{l}
w_{, y}-\beta_{y} \\
w_{, x}+\beta_{x}
\end{array}\right]
$$

As in the Krichhoff plate theory, the state of stress in
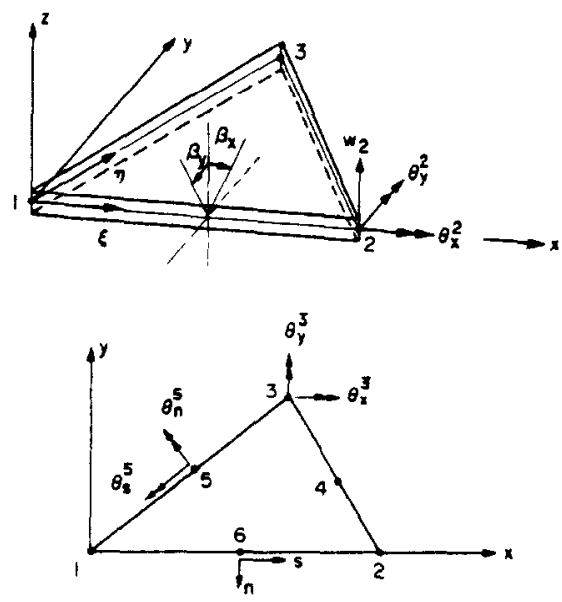

$$
\begin{aligned}
& \text { BENOING }
\end{aligned}
$$

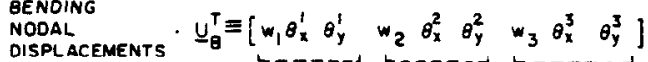

$$
\begin{aligned}
& \text { NOOE }{ }^{-1} \text { NOOE } 2 \text { NOOE } 3
\end{aligned}
$$

Fig. 2. Notation used in calculation of bending stiffness matrix. the plate is defined with plane stress assumption, i.e. $\sigma_{z}=0$. The stress-strain relations for a particular layer are:

$$
\sigma_{b}=\left[\begin{array}{l}
\sigma_{x} \\
\sigma_{y} \\
\tau_{x y}
\end{array}\right]=z C_{x}
$$

and

$$
\sigma_{s}=\left[\begin{array}{c}
\tau_{y z} \\
\tau_{z x}
\end{array}\right]=C_{s} \gamma
$$

where the matrices $\mathbf{C}$ and $C_{s}$ are the elasticity matrices in plane stress and shear deformations, respectively $[1,2]$.

With the kinematics as given by eqns (1)-(4) and the material descriptions given by eqns (5) and (6) the strain energy is

$$
q=q_{b}+q_{s}
$$

where

$$
\begin{aligned}
& \mathscr{U}_{b}=\frac{1}{2} \int_{A} \kappa^{T} \mathrm{D}_{b} \kappa \mathrm{d} A \\
& \mathscr{U}_{s}=\frac{1}{2} \int_{A} \gamma^{T} \mathrm{D}_{s} \gamma \mathrm{d} A
\end{aligned}
$$


and

$$
\begin{aligned}
& \mathbf{D}_{b}=\int_{-h / 2}^{h / 2} \mathrm{C} z^{2} \mathrm{~d} z \\
& \mathbf{D}_{s}=k \int_{-h / 2}^{h / 2} C_{s} \mathrm{~d} z .
\end{aligned}
$$

The variables $\mathscr{U}_{b}$ and $u_{s}$ represent the bending and transverse shear contributions, respectively, and $k$ in eqn (11) is a shear correction factor to account for the non-uniformity of the transverse shear stresses through the plate thickness.

In eqns (8) and (9) the matrices $D_{b}$ and $D_{s}$ are functions of the thickness of the plate, $h$, and of the elastic properties of the different layers; the variable $A$ is the area of the middle surface of the plate. For the important practical case of an isotropic homogeneous plate of constant thickness we have

$\mathrm{D}_{b}=\frac{E h^{3}}{12\left(1-\nu^{2}\right)}\left[\begin{array}{ccc}1 & \nu & 0 \\ & 1 & 0 \\ (\mathrm{sym}) & \frac{1-\nu}{2}\end{array}\right]: \mathrm{D}_{s}=\frac{E h k}{2(1+\nu)}\left[\begin{array}{ll}1 & 0 \\ 0 & 1\end{array}\right]$.

The variables $E$ and $\nu$ in eqn (12) are the Young's modulus and Poisson's ratio, and $k$ is usually taken as $5 / 6$.

By definition, the bending moments $M$ and shear forces $\mathbf{Q}$ are obtained by integration of the stresses through the thickness:

$$
\mathbf{M}=\left[\begin{array}{c}
M_{x} \\
M_{y} \\
M_{x y}
\end{array}\right]=\int_{-h / 2}^{h / 2} \sigma z \mathrm{~d} z=\mathrm{D}_{b} \kappa
$$

and

$$
Q=\left[\begin{array}{l}
Q_{y} \\
Q_{x}
\end{array}\right]=k \int_{-h / 2}^{h / 2} \sigma_{s} \mathrm{~d} z=\mathbf{D}_{s} \gamma .
$$

For thin plates the transverse shear strains are small and therefore the transverse shear strain energy $u_{s}$ is negligible compared to the bending energy. The finite element model based on the energies in eqns (7)-(9) must be able to represent this constraint. Therefore, the stiffness matrix of the element for the analysis of thin plates is based only on the expression,

$$
q=\frac{1}{2} \int_{A} \kappa^{T} \mathbf{D}_{b} \times \mathrm{d} A
$$

and this results in a discrete-Kirchhoff-theory (DKT) element formulation.

Equation (15) contains only the first derivatives of $\beta_{x}$ and $\beta_{y}$ and hence it is reiatively easy to establish interpolation functions that satisfy the compatibility requirements. However, since $\beta_{x}, \beta_{y}$ are the only variables in eqn (15), it is necessary to relate the rotations of the normal to the middle surface to the transverse displacement $w$ (which does not appear in eqn (15)). We use that $\beta_{x}$ and $\beta_{y}$ vary quadratically over the element, i.e.

$$
\beta_{x}=\sum_{i=1}^{6} h_{i} \theta_{y}^{i} ; \beta_{y}=\sum_{i=1}^{6} h_{1} \theta_{x}^{i}
$$

where $\theta_{x}{ }^{i}$ and $\theta_{y}{ }^{i}$ are the nodal values at the corners and at the mid-points of the sides (see Fig. 2), and then we used the following constraints:

(1) the Kirchhoff hypothesis is imposed at:

(a) the corner nodes

$$
\gamma=\left[\begin{array}{l}
w_{, y}-\beta_{y} \\
w_{x}+\beta_{x}
\end{array}\right]=0 \text { at nodes } 1,2 \text { and } 3
$$

(b) the mid-points of the sides (defined anticlockwise around the element boundary),

$$
w_{, s}-\beta_{s}=0 \quad k=4,5,6 ;
$$

(2) the variation of $w$ along the sides is cubic, i.e.

$$
\underset{\text { at node } k}{w, s}\left|=-\frac{3}{2 l_{i j}} w_{i}-\frac{1}{4} w_{, s}\right|+\frac{3}{2 l_{i j}} w_{r}-\left.\frac{1}{4} w_{\text {at node } i}\right|_{\text {at node }}
$$

with $k$ denoting the mid-node of side $i j$ and $l_{i j}$ equal to the length of the side $i j$; and

(3) a linear variation of $\beta_{n}$ is imposed along the sides, i.e.

$$
\beta_{n}{ }^{k}=\frac{1}{2}\left(\beta_{n}{ }^{i}+\beta_{n}{ }^{i}\right)
$$

where $k=4,5,6$ denotes the mid-points of the element sides. Using eqns (16)-(20) we obtain [8]

$$
\begin{aligned}
& \beta_{x}=\mathbf{H}_{x}{ }^{T}(\xi, \eta) \mathbf{U}_{B} \\
& \beta_{y}=\mathbf{H}_{y}{ }^{T}(\xi, \eta) \mathbf{U}_{B}
\end{aligned}
$$

where the $\mathbf{H}_{x}$ and $\mathbf{H}_{y}$ are nine component vectors of new shape functions, and $\xi, \eta$ are the natural elements coordinates.

The evaluation of the stiffness matrix now follows the standard procedures of the finite element displacement method. We have

$$
\boldsymbol{c}=\mathbf{B}_{B} \mathbf{U}_{B}
$$

where $B_{B}$ is the strain-displacement transformation matrix,

$$
\mathbf{B}_{B}(\xi, \eta)=\frac{1}{2 A}\left[\begin{array}{c}
y_{3} \mathbf{H}_{x, \xi}^{T} \\
-x_{3} \mathbf{H}_{y, \xi}^{T}+x_{2} \mathbf{H}_{y, \eta}^{T} \\
-x_{3} \mathbf{H}_{x, \xi}^{T}+x_{2} \mathbf{H}_{x, \eta}^{T}+y_{3} \mathbf{H}_{y, \xi}^{T}
\end{array}\right] .
$$

The bending stiffness matrix of the element is then

$$
\mathbf{K}_{B}=2 A \int_{0}^{1} \int_{0}^{1-\eta} B_{B}^{T} \mathbf{D}_{b} \mathbf{B}_{B} \mathrm{~d} \xi \mathrm{d} \eta
$$

If the thickness and the material properties are constant over the element, the exact integration of $\mathbf{K}_{B}$ is obtained using three numerical integration points located at the mid-points of the edges or inside because the integral involves only quadratic terms.

Once the nodal displacements have been calculated, the bending moments $M$ at any point in the element can be obtained

$$
\mathbf{M}(\xi, \eta)=\mathbf{D}_{b} \mathbf{B}_{B}(\xi, \eta) \mathbf{U}_{B}
$$


where

$$
\begin{aligned}
& x=\xi x_{2}+\eta x_{3} \\
& y=\eta y_{3} .
\end{aligned}
$$

\subsection{In-plane rotation stiffness}

The superposition of $\mathbf{K}_{M}$ and $\mathbf{K}_{B}$ in a three-dimensional space yields an element stiffness matrix that in a local coordinate system has zero in-plane rotational stiffness. The value of $K_{\theta_{2}}$ equal to $10^{-4}$ times the smallest bending stiffness is thus added into this degreeof-freedom to obtain 6 stiffness degrees-of-freedom per node. This value is chosen to remove the in-plane rotational singularity from the element stiffness matrix when the local $x-y-z$ axes coincide with the global $X-Y \cdot Z$ axes.

\section{FORMULATION FOR LARGE DISPLACEMENT ANALYSIS}

The formulation of the element for large displacement analysis follows closely the developments presented in [10]. In that work we studied an updated and a total Lagrangian formulation of a beam element that was based on Hermitian interpolation of the bending displacements and linear interpolation of the longitudinal and torsional displacements. We concluded in that study that the use of both formulations yields in an analysis identically the same numerical results, but that the updated Lagrangian formulation requires less computations. For the same reasons, considering the 3-node shell element, which is also based on different interpolations for the bending and membrane displacements, an updated Lagrangian formulation will be more effective.

\subsection{Governing equilibrium equations}

The application of the principle of virtual displacements to an element yields in the incremental large displacement analysis [2]

$$
\left({ }_{i}^{t} K_{L}+{ }_{t}^{t} K_{N L}\right) \Delta U^{(i)}={ }^{t+\Delta t} R-{ }_{t+\Delta t}^{t+\Delta t} F^{(i-1)}
$$

where ${ }_{t}^{t} \mathbf{K}_{L}$ and ${ }_{t}^{t} \mathbf{K}_{N L}$ are the linear and nonlinear strain stiffness matrices, ${ }^{t+\Delta t} \mathbf{R}$ is the load vector corresponding to the external loads at time $t+\Delta t,{ }_{t+\Delta t}^{t+\Delta_{t}} \mathrm{~F}^{(1)}$ is a vector of nodal point forces corresponding to the element stresses at time $t+\Delta t$ and iteration $(i-1)$, and

$$
{ }^{t+\Delta t} U^{(i)}={ }^{t+\Delta t} U^{(i-1)}+\Delta U^{(i)} .
$$

In eqn (27) we consider the equilibrium equations of a single element from which the equilibrium equations of an assemblage of elements can be obtained using standard procedures [2]. Also, the updated Lagrangian formulation is used because the reference configuration (denoted by left subscripts) corresponds to the times at which the quantities are calculated (denoted by the left superscripts).

Figure 3 shows schematically an element undergoing large displacements, and defines the coordinate systems and displacements used in the calculation of the stiffiness matrix and force vector. We note that the global coordinate system $X, Y, Z$ is stationary and that the total and incremental nodal point displacements and rotations are evaluated in this coordinate system. In addition, we consider body-attached coordinate systems. The stiffness

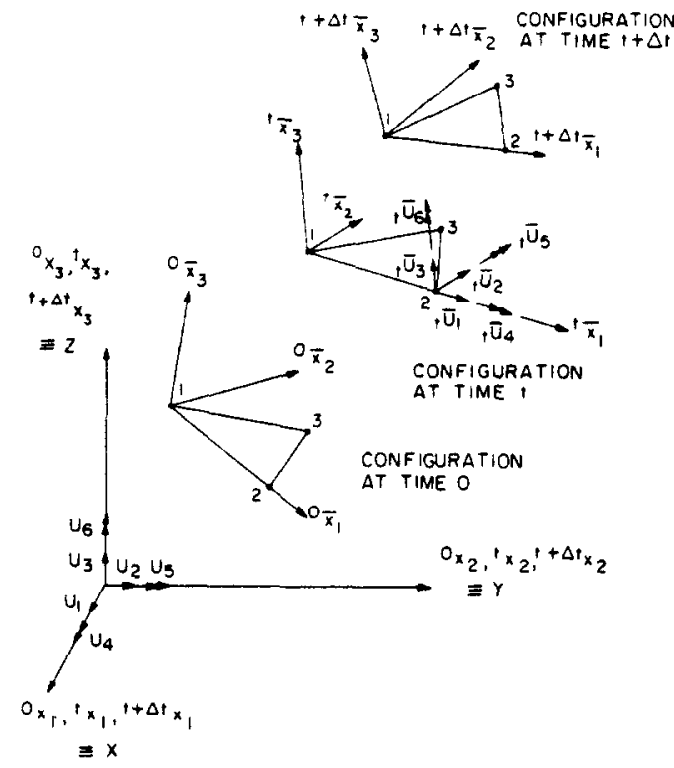

Fig. 3. Large displacement motion of the element.

matrix and force vector of the element corresponding to the global stationary coordinate system are evaluated by calculating these matrices first in the current bodyattached coordinate frame. Since small strain but large rotation conditions are assumed, the membrane and bending contributions can-in the body-attached coordinate system-be evaluated separately much in the same way as in linear analysis. The matrices are then transformed to the global coordinate frame. In the following sections we, therefore, only need to discuss how the bending and membrane stiffness matrices and the corresponding nodal point force vectors are calculated in the body-attached coordinate system.

\subsection{Membrane force vector and stiffness matrix}

The membrane displacements at any time $\tau$ yield the membrane forces corresponding to that time. Let ${ }^{~} \overline{\mathrm{U}}_{M}$ be the total membrane displacements at time $\tau$ in the plane of the element as shown in Fig. 4. These displacements are calculated from the difference in the local element coordinates at times equal to $\tau$ and zero. The element internal membrane forces, $\mathrm{N}$, are then calculated as in linear analysis. Using the strain-displacement matrix corresponding to the original element configuration,

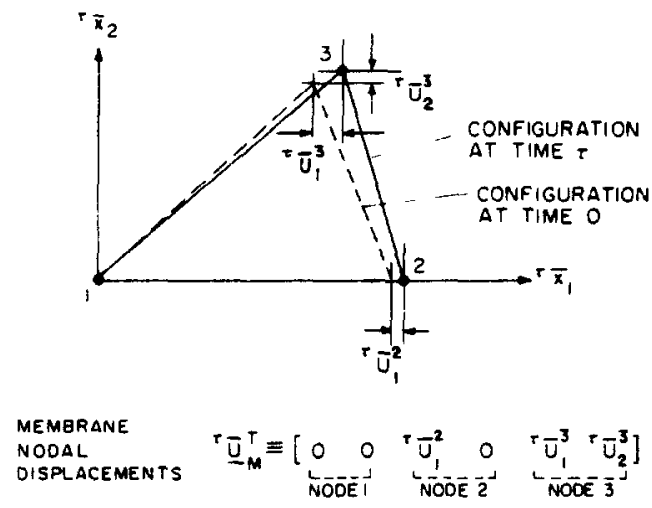

Fig. 4. Element membrane displacements. 
$\mathbf{B}_{M}$, and the displacements shown in Fig. 4, we have

$$
{ }^{\top} \overline{\mathbf{N}}=h \mathbf{C} \mathbf{B}_{M}{ }^{\top} \overline{\mathrm{U}}_{M}
$$

where the bar over a quantity signifies that it is defined in the local coordinate system.

The nodal point force vector corresponding to the internal membrane forces is then

$$
{ }_{\tau}^{\tau} \overline{\mathbf{F}}=\int_{A} \mathbf{B}_{M}^{T+} \overline{\mathbf{N}} \mathrm{d} A .
$$

The linear strain stiffness matrix in the local coordinate system is calculated as in linear analysis,

$$
{ }_{\tau}^{\tau} \overline{\mathbf{K}}_{L_{M}}=\overline{\mathbf{K}}_{M}
$$

and the nonlinear strain stiffness matrix is

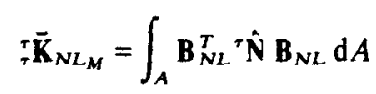

where $\mathbf{B}_{N L}$ and ${ }^{+} \hat{\mathbf{N}}$ are given in Appendix A.

\subsection{Bending force vector and stiffness matrix}

Whereas the membrane internal element forces are calculated from the total membrane displacements at time $\tau$, the element bending moments are calculated by incrementation, i.e. we have

$$
{ }^{\tau} \overline{\mathbf{M}}={ }^{r-\Delta t} \overline{\mathbf{M}}{ }_{{ }_{\tau}-\Delta r} \overline{\mathbf{M}} .
$$

The incremental bending moments are obtained from the local curvature increments,

$$
\begin{array}{rr}
\tau-\Delta t \overline{\boldsymbol{K}}=B_{B} & \tau-\Delta t \overline{\mathbf{U}}_{B} \\
\tau-\Delta t \overline{\mathbf{M}}=\mathrm{D}_{B} & \tau-\Delta t \overline{\boldsymbol{K}}
\end{array}
$$

where $\mathbf{B}_{B}$ is the constant generalized strain-displacement matrix defined in eqn (23), and ${ }_{+-\Delta t} \overline{\mathrm{U}}_{B}$ is a vector storing the bending displacement increments from the configuration at time $\tau-\Delta t$.

The nodal point force vector corresponding to the element bending moments is then

$$
{ }_{\tau}^{\tau} \overline{\mathrm{F}}_{B}=\int_{A} \mathbf{B}_{B}{ }^{T} \tau \overline{\mathrm{M}} \mathrm{d} A
$$

Considering the element stiffness matrix, the linear strain stiffness matrix is evaluated as in linear analysis

$$
\tau_{\tau}^{\tau} \overline{\mathbf{K}}_{L_{B}}=\cdot \mathbf{K}_{B}
$$

and the effect of the nonlinear strain stiffness matrix is neglected. This assumption is appropriate because the bending effect is relatively small, and only an approximate stiffness matrix is required in the analysis.

The above calculations (eqns (33)-(35)) assume that the incremental displacements are small, and the bending distortions of the element can be neglected in the evaluation of the generalized strain-displacement matrix.

\section{SAMPLE SOLUTIONS}

To indicate the applicability and effectiveness of the element we present in this section the results obtained in
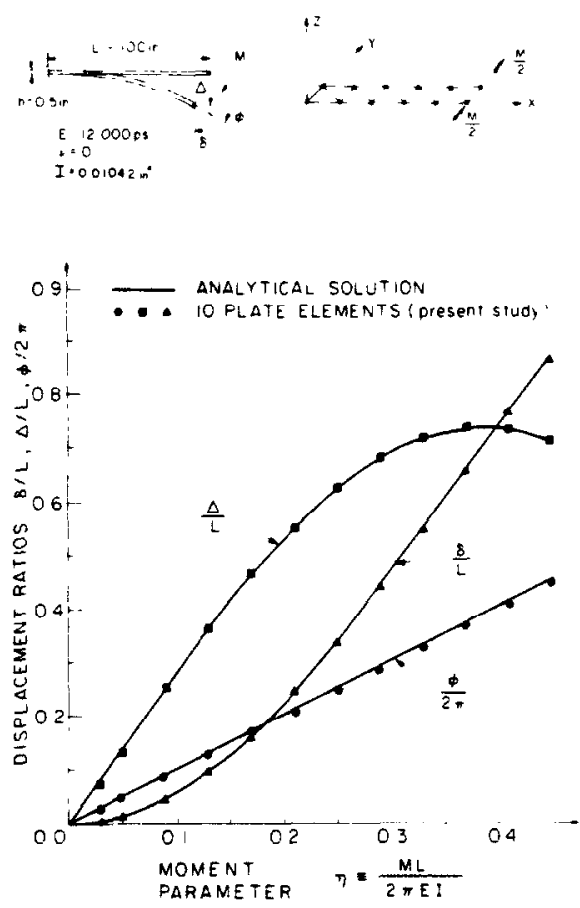

Fig. 5. Large deflection response of a cantilever.

the analyses of some problems. Tests of the element in the linear analysis of plates have been presented in [8].

\subsection{Large deflection and rotation analysis of a cantilever}

The cantilever shown in Fig. 5 was analyzed for its large deflection response. Five layers of elements were used in the analysis. This is a problem that is frequently used to study the characteristics of an element $[6,10]$.

Figure 5 shows the predicted response in the analysis and the response calculated analytically. We observe good correspondence between the two solutions.

\subsection{Analysis of a pinched cylindrical shell}

The structure analyzed and a typical finite element idealization used are shown in Fig. 6. In the figure the $10 \times 10$ mesh used is shown, but the analysis was also carried out with $4 \times 4,6 \times 6,8 \times 8$ and $16 \times 16$ mesh topologies. Figures 7-9 give calculated displacement and stress resultant distributions along the lines DC, BC and $A D$ of the shell, respectively. It is seen that the finite element predictions converge rapidly to the analytical solution [11] as a reasonable number of shell elements is employed in the structural idealization. Table 1 sum-

Table 1. Total solution times in analysis of pinched cylindrical shell (on a CDC Cyber 175)

\begin{tabular}{|c|c|}
\hline Grid & Solution time (sec.) \\
\hline $4 \times 4$ & 1.00 \\
\hline $6 \times 6$ & 1.86 \\
\hline $8 \times 8$ & 3.19 \\
\hline $10 \times 10$ & 5.73 \\
\hline $16 \times 16$ & 21.94 \\
\hline
\end{tabular}




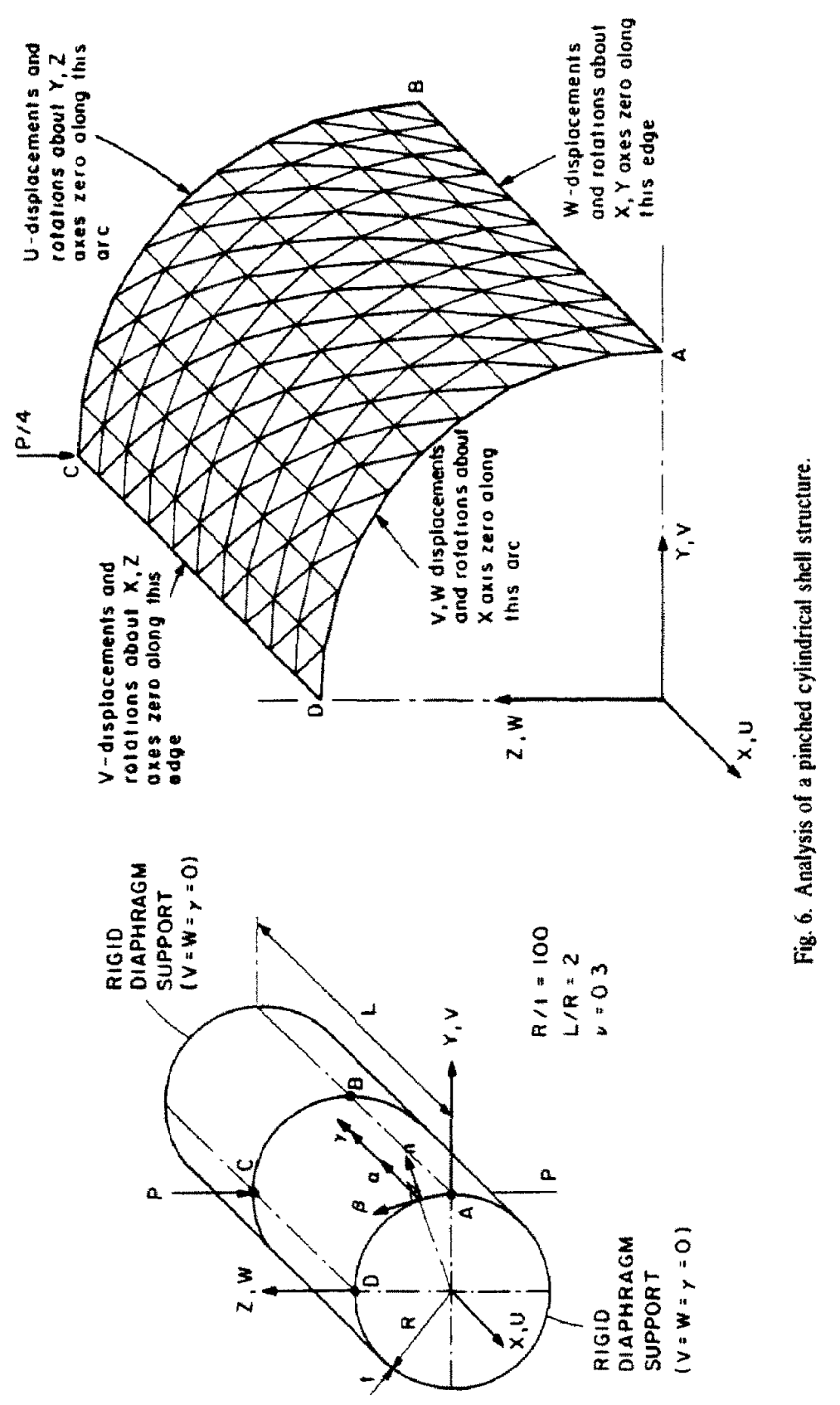



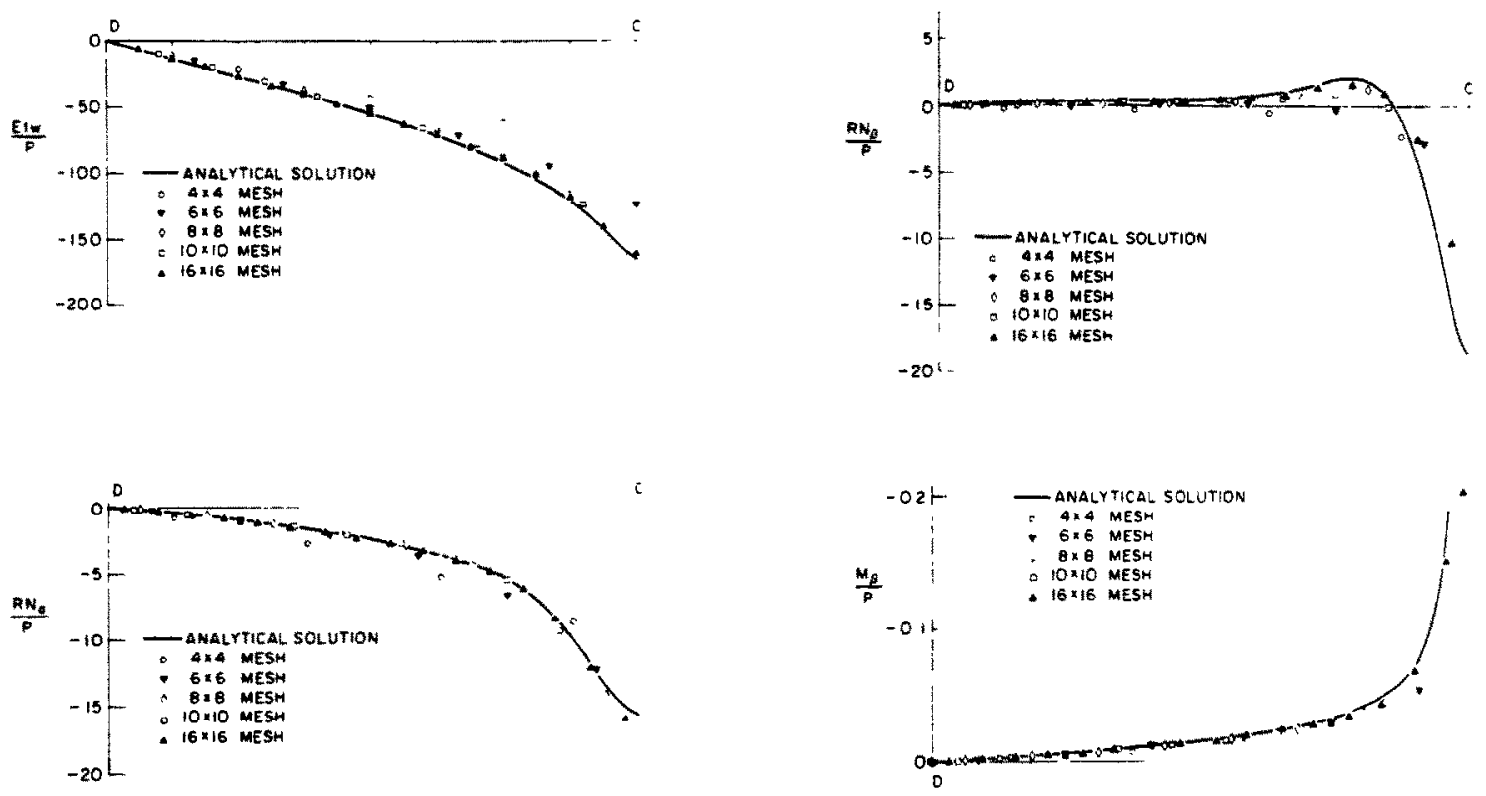

Fig. 7. Predicted displacement and stress distributions along DC of shell in Fig. 6 .
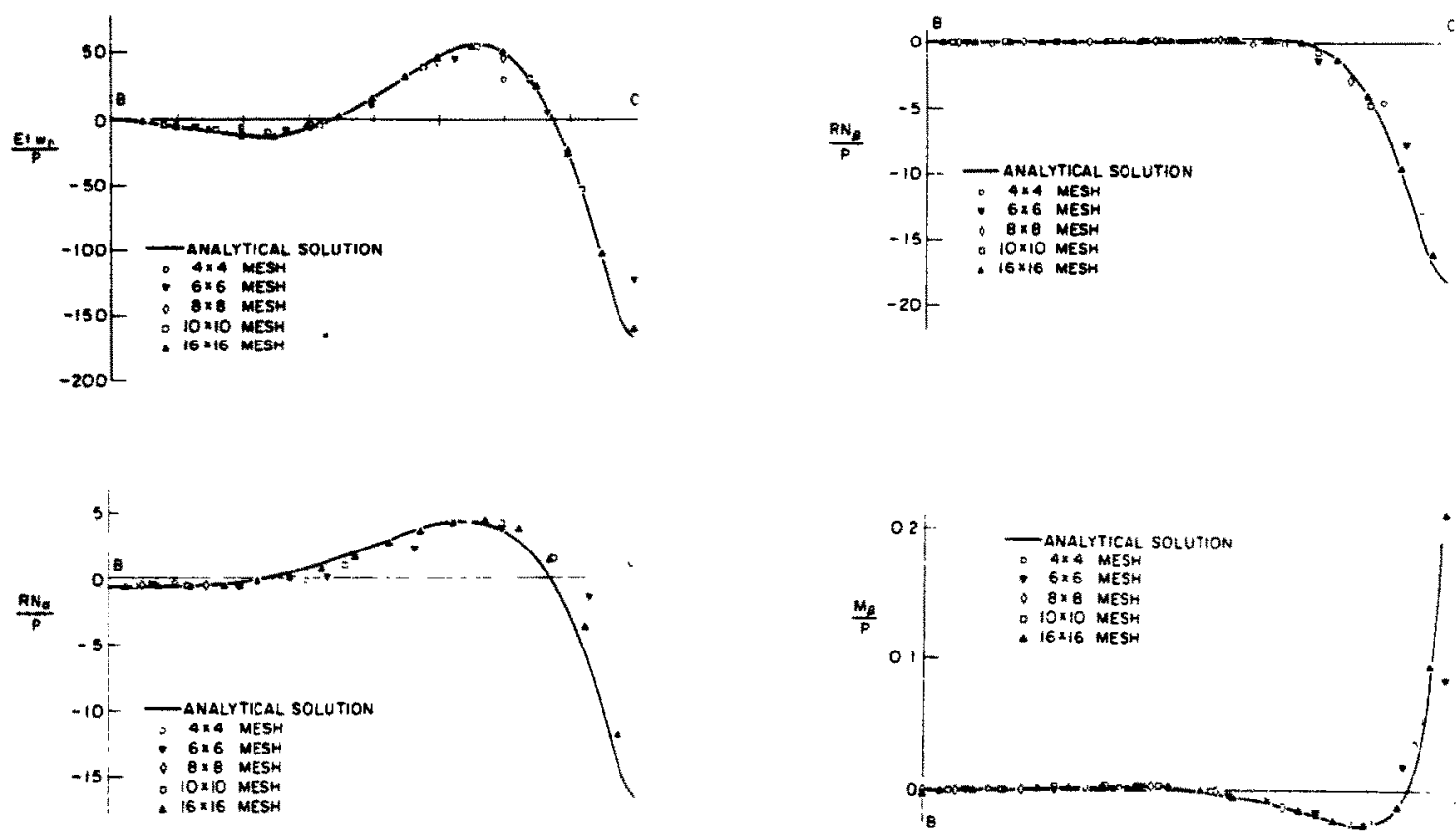

Fig. 8. Predicted displacement and stress distributions along BC of shell in Fig. 6.

marizes the solution times used in the analysis of the shell structure.

\subsection{Large displacement analysis of a simply-supported} plate

The plate was subjected to a uniform pressure loading q. Figure 10 shows the finite element idealization used for the plate and the response predicted. In a first analysis, the edges were constrained not to move in the plane of the plate, and in a second analysis, the $u$ and $v$ edge dis- placements were left free. The predicted responses in these analyses are compared in Fig. 10 with other solutions reported earlier $[12,3]$.

\subsection{Large displacement analysis of a spherical shell}

The shell described in Fig. 11 was analyzed for its large displacement response using a $5 \times 5$ mesh. Figure 12 shows the response predicted when the shell is subjected to the concentrated apex load. The post-buckling response was calculated by adding a stiffness of $12,000 \mathrm{lb} / \mathrm{in}$ 

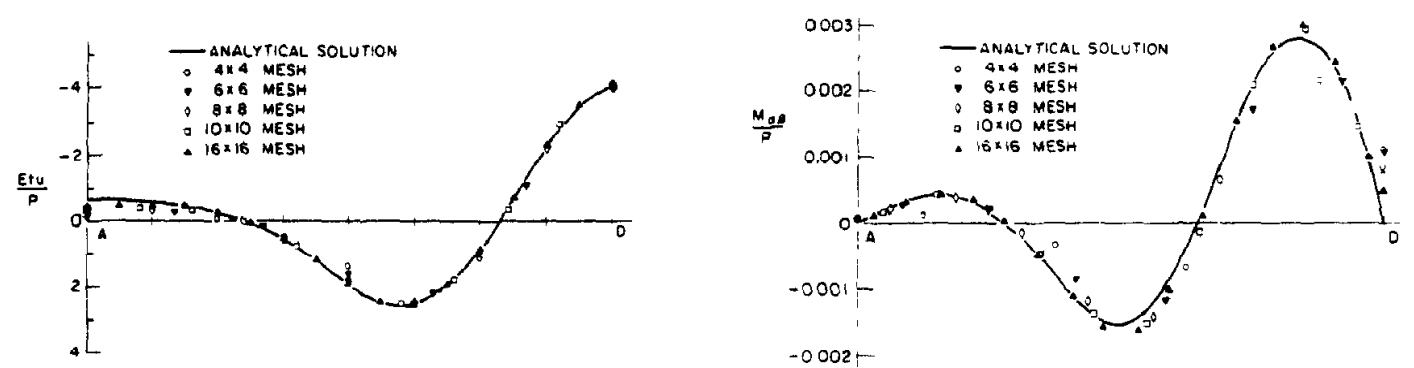

Fig. 9. Predicted displacement and stress distributions along AD of shell in Fig. 6.

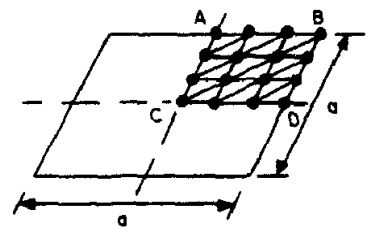

$E=$ YOUNE'S MODULUS $=10^{7}$ PSI

$v=$ POISSONSRATIO $=\sqrt{0.1}$

$h=$ THICKNESS $=0.12 \mathrm{IN}$

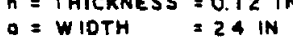

$0=$ WIOTH 224 IN
$q=$ UNIFOAM APPLIEO PRESSURE

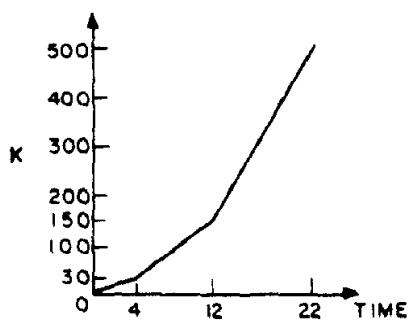

APPLIED PRESSURE LOAD

ALL EDGES ARE SIMPLY-SUPPORTEO

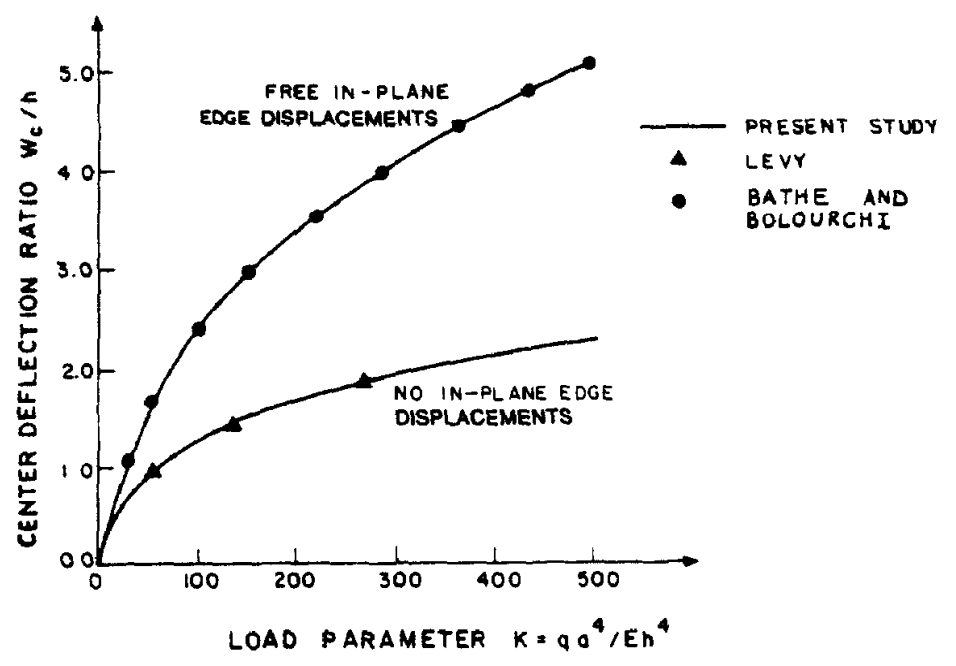

Fig. 10. Analysis of a simply-supported plate.

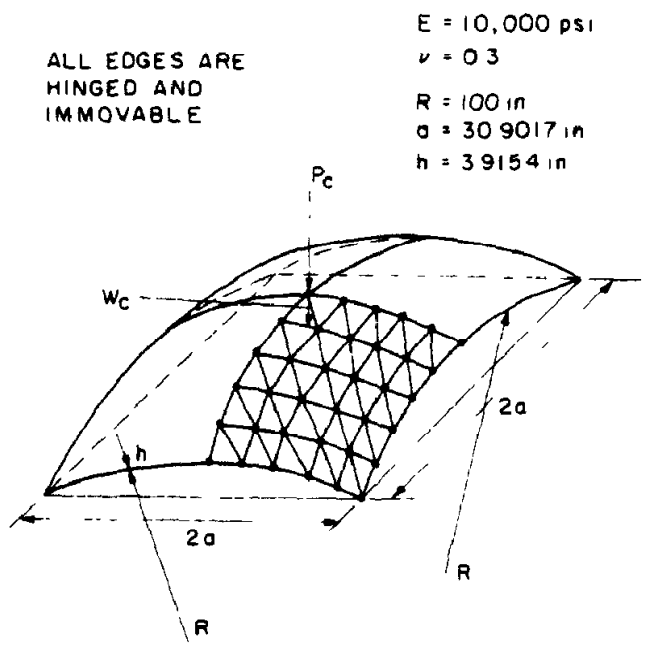

Fig. 11. Spherical shell subjected to apex load.

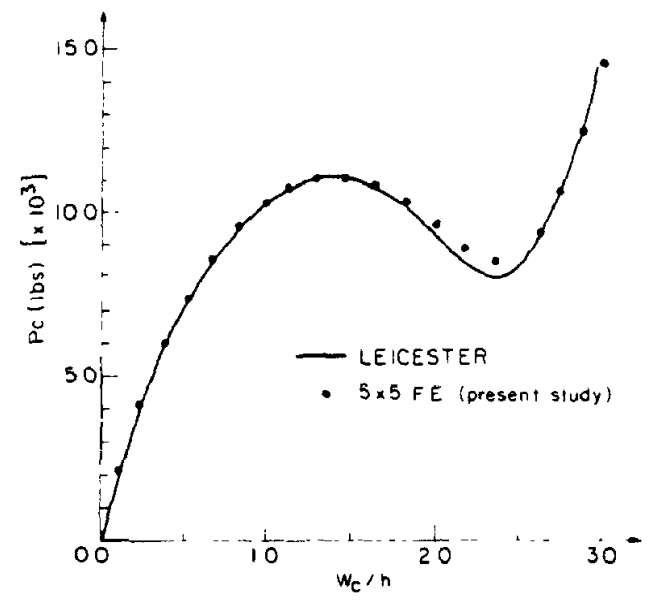

Fig. 12. Displacement response of spherical shell in Fig. 11. 
to the $w_{c}$ degree-of-freedom. This stiffness renders the stiffness matrix of the total structure positive-definite throughout the response history.

The result obtained in the finite element analysis is compared in Fig. 12 with the solution reported by Leicester $[13,14]$.

\section{CONCLUDING REMARKS}

The objective in this paper was to present a simple and effective 3-node triangular plate/shell element. The element is easy to use and very versatile. It is also reliable because the element stiffness matrix is integrated exactly: hence, the element contains no spurious zero energy modes. The element has excellent bending characteristics but a shortcoming is that the membrane forces are assumed to be constant. Therefore, it is still desirable to improve the element membrane behavior.

We have tested the element in various applications and some solution results are presented in the paper. Our experiences with the element show that it complements in a very effective way the higher-order isoparametric shell elements also available in ADINA.

Acknowledgement-We gratefully acknowledge the financial support of the ADINA users group.

\section{REFERENCES}

1. 0. C. Zienkiewicz, The Finite Element Method. McGrawHill, New York (1977).

2. K. J. Bathe, Finite Element Procedures in Engineering Analysis, Prentice-Hall, Englewood Cliffs, New Jersey, in press.

3. K. J. Bathe and S. Bolourchi, A geometric and material nonlinear plate and shell element. Comput. Structures 11, 23-48 (1979).

4. T. J. R. Hughes, R. L. Taylor and W. Kanoknukulchai, A simple and efficient finite element for plate bending. Int. $J$. Numer. Meth. Engng 11, 1529-1543 (1977).

5. O. C. Zienkiewicz. J. Bauer, K. Morgan and E. Onate, A simple and efficient element for axisymmetric shells. Int. $J$. Numer. Meth. Engng 11, 1545-1558 (1977).
8. J. L. Batoz, K. J. Bathe and L. W. Ho, A study of three-node triangular plate bending elements. Int. J. Numer. Meth. Engng, 15, 1771-1812 (1980).

9. R. W. Clough and E. L. Wilson, Dynamic finite element analysis of arbitrary thin shells. Comput. Structures 1, 33-56 (1971).

10. K. J. Bathe and S. Bolourchi, Large displacement analysis of three-dimensional beam structures. Int. J. Numer. Meth. Engng 14, 861-986 (1979).

11. G. M. Lindberg, M. D. Olson and G. R. Cowper, New developments in the finite element analysis of shells. National Research Council of Canada, Quart. Bull. Div. Mech. Engng and the National Aeronautical Establishment, Vol. 4, pp. 1-38, 1969.

12. S. Levy, Bending of rectangular plates with large deflections. Tech. Notes, NACA, No 846 (1942).

13. R. H. Leicester, Finite deformations of shallow shells. Proc. ASCE 94(EM6), 1409-1423 (1968).

14. G. S. Dhatt, Instability of thin shells by the finite element method. IASS Symp. for Folded Plates and Prismatic Structures, Vienna. 1970.

\section{APPENDIX A}

Calculation of nonlinear strain stiffness matrix

Let ${ }^{\prime} \bar{N}_{3},{ }^{\prime} \bar{N}_{2}$ and ${ }^{\top} \bar{N}_{12}$ be the membrane force components given in eqn (29), and

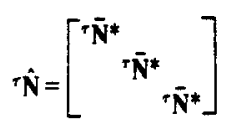

where

$$
{ }^{\top} \overline{\mathbf{N}}^{*}=\left[\begin{array}{cc}
{ }^{\top} \bar{N}_{1} & { }^{\top} \bar{N}_{12} \\
{ }^{\top} \bar{N}_{12} & { }^{\prime} \bar{N}_{2}
\end{array}\right]
$$

Then corresponding to the displacement vector ' $\overline{\mathbf{U}}$,

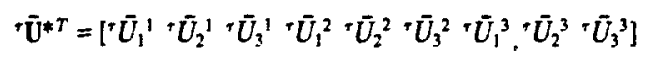

we have

$$
\mathbf{B}_{N L}=\frac{1}{2 A}\left[\begin{array}{ccccccccc}
-\bar{x}_{2}{ }^{3} & 0 & 0 & \bar{x}_{2}{ }^{3} & 0 & 0 & 0 & 0 & 0 \\
\bar{x}_{1}{ }^{32} & 0 & 0 & -\bar{x}_{1}{ }^{3} & 0 & 0 & \bar{x}_{1}{ }^{2} & 0 & 0 \\
0 & -\bar{x}_{2}{ }^{3} & 0 & 0 & \bar{x}_{2}{ }^{3} & 0 & 0 & 0 & 0 \\
0 & \bar{x}_{1}{ }^{32} & 0 & 0 & -\bar{x}_{1}{ }^{3} & 0 & 0 & \bar{x}_{1}{ }^{2} & 0 \\
0 & 0 & -\bar{x}_{2}{ }^{3} & 0 & 0 & \bar{x}_{2}{ }^{3} & 0 & 0 & 0 \\
0 & 0 & \bar{x}_{1}{ }^{32} & 0 & 0 & -\bar{x}_{1}{ }^{3} & 0 & 0 & \bar{x}_{1}{ }^{2}
\end{array}\right]
$$

6. J. H. Argyris, P. C. Dunne, G. A. Malejannakis and E. Schelkle, A simple triangular facet shell element with applications to linear and non-linear equilibrium and elastic stability problems. Comput. Meth. Appl. Mech. Engng 10. 371-403 (1977).

7. K. J. Bathe and L. W. Ho, Some results in the analysis of thin shell structures. In Nonlinear Finite Element Analysis in Structural Mechanics (Edited by W. Wunderlich et al.). Springer-Verlag, Berlin (1981). where $\bar{x}_{1}{ }^{k}$ is the $\bar{x}_{1}$-coordinate of node $k$ and

$$
\bar{x}_{1}{ }^{i j}=\bar{x}_{1}{ }^{\prime}-\bar{x}_{1}^{\prime}, \text { etc. }
$$

Note that the small strain assumption allows the following approximation:

$$
{ }^{\tau} \bar{x}_{1}^{k} \cong{ }^{0} \bar{x}_{1}{ }^{k} \equiv \bar{x}_{i}{ }^{k},
$$

that is, the undeformed local coordinates are used for all $\tau$. 